\title{
What are we curious about?
}

\section{Franziska Brändle ${ }^{1}$, Charley $M . \mathbf{W u}^{2}$, and Eric Schulz ${ }^{3, *}$}

\author{
${ }^{1}$ Max Planck Institute for Biological Cybernetics, Tübingen, Germany \\ ${ }^{2}$ Department of Psychology, Harvard University, Cambridge, MA \\ *Corresponding author: eric.schulz@tuebingen.mpg.de
}

\begin{abstract}
What are we curious about? Dubey and Griffiths propose a rational theory of curiosity that unifies previously contradictory novelty-based and complexity accounts. It also paves the way for future investigations, such as studying approximate models of curiosity as well as what causes abnormal levels of exploration.
\end{abstract}

Curiosity is ubiquitous in our daily lives, driving diverse behaviors such as reading books, visiting museums, or running experiments. But what does a formal theory of curiosity look like?

Existing theories coalesce around two categories. Novelty-based theories describe people as seeking out what they are least confident about, since novel stimuli offer the highest potential for gaining information ${ }^{1}$. Complexity theories predict people prefer exploring stimuli they are moderately confident about, as they offer the largest opportunities for improvement ${ }^{2}$. How can these seemingly opposing theories be reconciled?

Dubey and Griffiths ${ }^{3}$ present a rational theory of curiosity that unifies novelty-based and complexity theories as special cases. Casting curiosity as a mechanism enabling the maximization of future rewards, Dubey and Griffiths show how a rational agent should seek out stimuli (or tasks) that maximally change their overall value of knowledge $V$ :

$$
V=\sum_{k} p_{k} c_{k}
$$

The value of knowledge is a function of how often we encounter a task in the environment $p_{k}$ and our confidence $c_{k}$ (i.e., probability of success), summed up over all tasks $k$, under the assumption that an agent's confidence increases with exposure. To maximize future rewards, the agent needs to compute the rate at which the value of knowledge changes with respect to the exposure for any given task. Put simply, people should be curious about tasks that increase their ability to solve as many future tasks as possible. Curiosity can thus be expressed as a derivative (Fig. 1A) defining how much one expects to gain in their value of knowledge by performing a task.

This definition predicts that curiosity is shaped by the environment, specifically, by dependencies between the future probability of encountering a task $\left(p_{k}\right)$ and the number of previous encounters $\left(h_{k}\right)$. If past exposure and future occurrences are independent, the value of information is maximized by sampling the tasks corresponding to the lowest confidence - consistent with novelty-based theories of curiosity (Fig. 1B). For instance, when faced with an unpredictable environment, such as preparing for a trivia competition, you should prioritize learning what you are least confident about (e.g., brushing up on ancient Rome if you are generally poor at history).

However, in other cases there is structure to the environment, such that you are more exposed to stimuli you are confident about. If future occurrences are proportional to past exposure, the relation between curiosity and confidence is defined by an inverted U-shape - consistent with complexity theories (Fig. 1C). For example, if you are a tour guide in the city of Rome, learning answers to commonly asked questions would be more valuable than looking up facts about rarely visited buildings.

Dubey and Griffiths ${ }^{3}$ demonstrate how participants flexibly adapt their curiosity to the structure of the environment through an experiment based on trivia questions. Questions were either sampled randomly (independent condition) or proportionally (dependent condition) to the participants' a priori confidence ratings. Before the quiz, participants could reveal answers to questions, which were used as a measure of curiosity. As predicted, participants in the dependent condition were more curious about questions they rated as moderately confident, whereas participants in the independent condition were more curious about questions they rated as least confident (Fig. 1D). These results support the hypothesis that the relationship between curiosity and confidence is influenced by the structure of future occurrences.

Many theories assume curiosity leads to knowledge, which in turn will lead to rewards. Ideally, this means that curiosity can be incorporated into an agent's reward function, as proposed by Dubey and Griffiths. However, in most real world scenarios, one's confidence and the probability of occurrence for a given task are unknown and have to be inferred. This leads to some open empirical questions, two of which we discuss here. The first is to describe the sampling behavior of computationally tractable implementations of curiosity. The second is the explanation of when and how curiosity goes awry. 
A

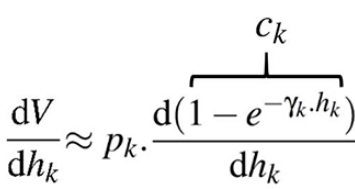

$V$ Value of knowledge

$k$ One of $n$ possible tasks

$h$ Exposure

$p$ Probability of occurrence

$c$ Confidence

$\gamma$ Learning rate
B

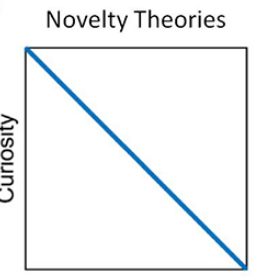

Confidence

C

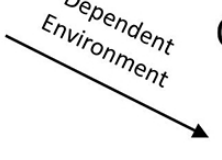

Complexity Theories

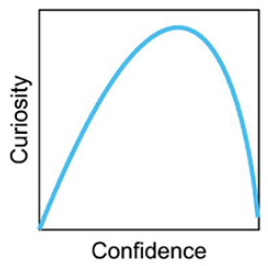

D

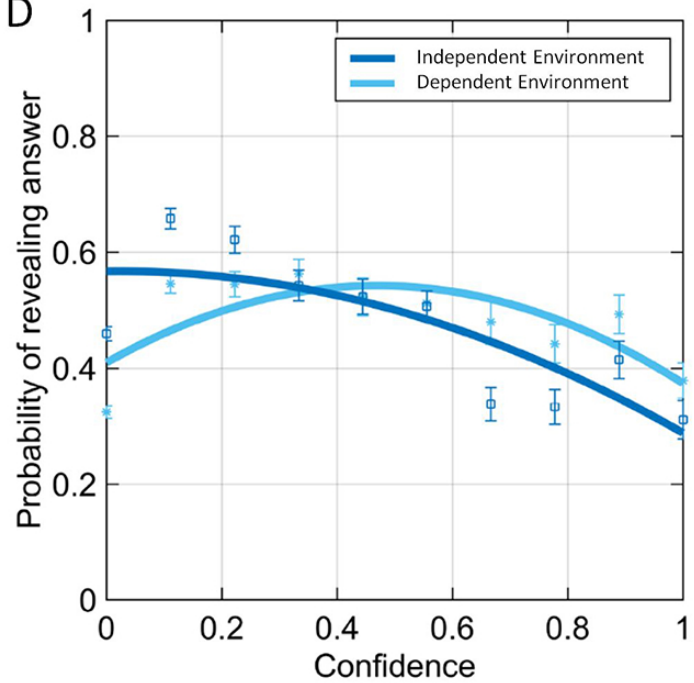

Figure 1. Different sampling patterns of curiosity. A: Dubey and Griffiths' theory of curiosity ${ }^{3}$. B: In independent environments, tasks are randomly sampled, i.e. independent of confidence. This creates a linear relation between confidence and curiosity. A rational agent should optimally sample tasks with the least confidence. C: In dependent environments, high confidence tasks are more likely to occur. Here, curiosity and confidence are related through an inverted U-shaped function and the agent should optimally sample tasks corresponding to moderate confidence. D: Experiment 1 from Dubey and Griffiths ${ }^{3}$, showing the relation between confidence and the probability of sampling a trivia question when manipulating the environment.

While Dubey and Griffiths' model is generally intractable in real world scenarios, several heuristics have been developed to approximate curiosity without considering future occurrences of stimuli.

One type of heuristics is uncertainty sampling ${ }^{4}$, which preferentially samples stimuli with the highest estimated uncertainty, taking confidence to be the inverse of uncertainty. Since new stimuli are typically more uncertain, this strategy mirrors novelty-based theories of curiosity and leads to a linear relation between confidence and curiosity.

Another type of heuristics maximizes expected model change ${ }^{5}$, by preferentially sampling stimuli that produce the largest expected improvement to the agent's predictive model of the environment. Since stimuli with intermediate level of confidence tend to maximize this improvement, this strategy mirrors complexity-based theories of curiosity and leads to an inverted U-shaped relation between confidence and curiosity.

The goal of the Dubey and Griffiths model is to maximize future rewards. However, the relationship between confidence and curiosity is also affected by how the agent balances acquiring useful information (exploration) with acquiring immediate rewards (exploitation). Several sampling strategies have been developed for such exploration-exploitation problems, where a prominent model of human behavior is upper confidence bound sampling (UCB). UCB combines the current reward expectation of a stimulus with an exploration bonus proportional to its estimated uncertainty ${ }^{6}$, thus integrating noveltybased curiosity with the goal of preferentially sampling highly-rewarding stimuli. Yet in practice, UCB produces behavior resembling complexity-based theories of curiosity, since stimuli with intermediate levels of confidence tend to have the highest combination of expected rewards and uncertainty. This suggests complexity-based sampling strategies can be difficult to distinguish from strategies that combine novelty-based curiosity with the goal of acquiring immediate rewards (see https://github.com/ericschulz/curiositysim for simulations). Future experiments should attempt to further tease apart theories of curiosity in more complex tasks.

Finally, there are cases where curiosity seemingly goes awry. For example, Kobayashi et al. ${ }^{7}$ showed that people sometimes enjoy savoring good outcomes rather than gathering the most useful information. Indeed, people sometimes deliberately ignore information, for example knowing about events that could elicit negative feelings, like death or divorce ${ }^{8}$. Another case of misdirected curiosity is over-exploration, which can be symptomatic of Obsessive Compulsive Disorder ${ }^{9}$. Dubey and Griffiths propose suboptimal behaviors arise from miscalculations of the model's parameters. However, further research is needed to understand the problem of inferring parameters from sparse data, and the implications for a mismatch between one's model and the environment ${ }^{10}$.

In summary, Dubey and Griffiths propose a rational account of curiosity that elegantly reconciles past theories of human curiosity. Moving forwards, algorithmic approximations of curiosity for boundedly rational agents and understanding the 
mechanisms underlying seemingly suboptimal levels of curiosity remain exciting questions for future investigations.

\section{References}

1. Daniel E. Berlyne. Novelty and curiosity as determinants of exploratory behaviour. British Journal of Psychology. General Section, 41(1-2):68-80, 1950.

2. Celeste Kidd, Steven T. Piantadosi, and Richard N. Aslin. The Goldilocks effect: Human infants allocate attention to visual sequences that are neither too simple nor too complex. PLOS ONE, 7(5):1-8, 2012.

3. Rachit Dubey and Thomas L. Griffiths. Reconciling novelty and complexity through a rational analysis of curiosity. Psychological Review.

4. David D Lewis and William A Gale. A sequential algorithm for training text classifiers. In SIGIR'94, pages 3-12. Springer, 1994.

5. Burr Settles. Active learning literature survey. Technical report, University of Wisconsin-Madison Department of Computer Sciences, 2009.

6. Eric Schulz and Samuel J Gershman. The algorithmic architecture of exploration in the human brain. Current Opinion in Neurobiology, 55:7-14, 2019.

7. Kenji Kobayashi, Silvio Ravaioli, Adrien Baranès, Michael Woodford, and Jacqueline Gottlieb. Diverse motives for human curiosity. Nature Human Behaviour, 3(6):587-595, 2019.

8. Gerd Gigerenzer and Rocio Garcia-Retamero. Cassandra's regret: The psychology of not wanting to know. Psychological Review, 124(2):179-196, 2017.

9. Tobias U Hauser, Michael Moutoussis, Reto Iannaccone, Silvia Brem, Susanne Walitza, Renate Drechsler, Peter Dayan, and Raymond J Dolan. Increased decision thresholds enhance information gathering performance in juvenile obsessivecompulsive disorder (OCD). PLoS Computational Biology, 13(4):e1005440, 2017.

10. Charley M Wu, Eric Schulz, Maarten Speekenbrink, Jonathan D Nelson, and Björn Meder. Generalization guides human exploration in vast decision spaces. Nature Human Behaviour, 2(12):915-924, 2018. 